\title{
Análise Multivariada do Processo de Evapotranspiração em Áreas de Cerrado e Cana-de-Açúcar
}

\author{
Anderson Luis Ruhoff, Claudinéia Brazil Saldanha, Walter Collischonn \\ Institu to de Pesquisas H idráulicas -IPH / UFR GS \\ anderson.ruhoff@ufrgs.br, collischonn@iph.ufrgs.br \\ Cintia Bertacchi U vo \\ Water Resources Engineering - L und U niversity \\ cintia.uvo@tvrl.Ith.se \\ Humberto Ribeiro da Rocha \\ Institu to de Astron omia, Geofísica e Ciências A tmosféricas -IA G / USP \\ humberto@model.iag.usp.br \\ O svaldo Machado Rodrigues Cabral \\ Cen tro Nacional de Pesquisa de M onitoramento por Satélite -Embrapa M eio Ambiente \\ ocabral@en pm.embrapa.br
}

Recebido: 06/03/09 - revisado: 16/06/09 - aceito: 07/12/09

\begin{abstract}
RESUMO
A evapotranspiração é uma das variáveis mais importantes do ciclo hidrológico, consistindo na ligação en tre en ergia, clima e disponibilidade hídrica. É considerado um fenômeno bastante complexo, pois depende da interação en tre diversas variáveis climáticas e do tipo e estágio de desenvolvimento da vegetação. 0 objetivo deste trabalho foi a análise de um conjunto amostral de variáveis climáticas, coletadas em dois sítios de monitoramento de vórtices turbulentos in stalados em áreas de cultivos de cana-deaçúcar e remanescentes florestais de cerrado sensu stricto na bacia do rio Grande -SP, para identificar o comportamento de cada uma dessas variáveis climáticas everificar quais a presentam uma maior influên cia no processo de evapotranspiração. Para tanto, foram aplicadas técnicas de estatística multivariada de análise de componentes principais e agru pamento de variáveis. Concluiu-se que o processo de evapotranspiração, para a área estudada, é comandado por três componentes, que explicam 75 e $82 \%$ da variância dos dados em áreas de cerrado e cultivos de cana-deaçúcar, respectivamente: (1) variação da oferta de energia, representada principalmente pelas variáveis $\mathrm{Ki}$ e $\mathrm{Rn}$, (2) processo de circulação atmosférica local, represen tada pelas variáveis de velocidade do ven to e pressão atmosférica, que regulam o sistema de precipitação e as con diç̧ões de u midade do ar e do solo, e, (3) con diç̧̃es da vegetação, que apresentam modifica ções sign ificativas com a alternância de estações secas e úmidas.
\end{abstract}

Palavraschave Estatística M ultivariada, Evapotran spiração, Cerrado, Cana-deA çúcar.

\section{INTRODUÇÃO}

A evapotranspiração é uma das variáveis mais importantes do ciclo hidrológico, consistindo na ligação entre energia, clima e disponibilidade hídrica. Pode ser estimada por diversos métodos, como medições de vórtices turbulentos, lisímetros, técnicas de balanço de energia e balanço de água no solo e por equações baseadas em dados microclimáticos, como Pennman-Monteith (Allen et al, 1998). A evapotranspiração é um fenômeno bastante com- plexo, pois depende da interação entre diversas variáveis climáticas e do tipo e estágio de desenvolvimento da vegetação (Kumar et al, 2002).

Considerando a complexidade de estimativa do processo de evapotranspiração, foi analisado um conjunto amostral de variáveis climáticas, coletadas em dois sítios de monitoramento de vórtices turbulentos instalados em áreas de cultivos de cana-deaçúcar e remanescentes florestais de cerrado sensu stricto na bacia do rio Grande -SP, para identificar o comportamento de cada uma dessas variáveis climáticas e verificar quais apresentam uma maior 
influência no processo de evapotranspiração. Para tanto, foram aplicadas técnicas de estatística multivariada de análise de componentes principais e agrupamento de variáveis.

Esse estudo insere-se em uma proposta de calibração do modelo hidrológico MGB-IPH a partir de estimativas de evapotranspiração através do modelo SEBAL (Ruhoff et al, 2008; Ruhoff et al, 2009). Com o desenvolvimento de modelos hidrológicos distríbuidos, como o MGB-IPH (Collischonn 2001 e Collischonn e Tucci 2001), utilizado no entendimento do comportamento hidrológico de bacias hidrográficas, na previsão de vazões visando a operação de reservatórios hidroelétricos, na modelagem de qualidade de água e no processo de erosão e transporte de sedimentos, torna-se necessário a insersão de variáveis climáticas com boa precisão, de modo que o resultado dos processos de simulação hidrológica sejam coerentes com a realidade climática da área de estudo. Assim, o conhecimento do comportamento de cada uma das variáveis climáticas é fundamental para estimar o processo de evapotranspiração com precisão.

\section{ÁREA DE ESTUDO}

0 estudo foi realizada em uma área compreendida entre as coordenadas de $20^{\circ} 30^{\prime}$ e $22^{\circ} 30^{\prime}$ de latitude sul e $46^{\circ} 30^{\prime}$ e $48^{\circ} 30^{\prime}$ de longitude oeste. A área localiza-se no estado de São Paulo e é caracterizada pela intensa produção de cana-de-açúcar e pelos remanescentes florestais do Parque Estadual da Vassununga. Os sítios experimentais de monitoramento de fluxos turbulentos (figura 1) localizamse no Cerrado Pé-de-Gigante, um ecossistema natural de cerrado sensu stricto, em Santa Rita do Passa Quatro, e na U sina Santa Elisa, no agrossistema da cana-de-açúcar, em Sertãozinho.

A área de cerrado sensu stricto consiste em variações fisionômicas de (1) campos sujos, que se apresentam como gramíneas de até $0,5 \mathrm{~m}$, que ocupam encostas íngremes; (2) campos cerrados, que apresentam predomínio herbáceo-subarbustivo, com árvores esparsas de até $7 \mathrm{~m}$; (3) cerrado, composto principalmente por arbustos e árvores de até 5 m, em grande adensamento; (4) cerradão, caracterizado por florestas estacionais semideciduais, com predomínio de árvores de aproximadamente $10 \mathrm{~m}$ e componente herbáceo pouco desenvolvido (Batalha 1997). A figura 2 apresenta as variações fisionômicas do cerrado sen su stricto.

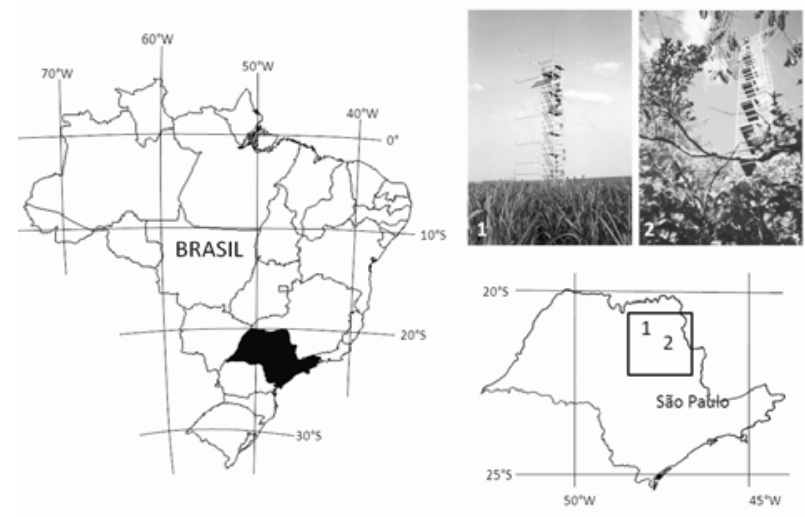

Figura 1 -Localização da área de estudo e dos sítios de monitoramento dos fluxos de vórtices turbulentos em plantações de cana-de-açúcar (1) e cerrado sensu stricto (2).

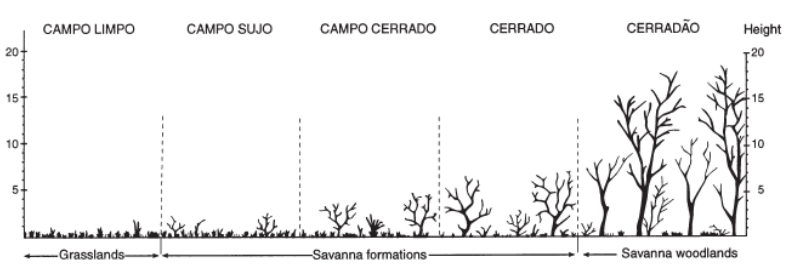

Figura 2 -Variações fisionômicas da vegetação em áreas de cerrado sensu strido. Fonte: Furley (1999).

Segundo estudos de Rocha et al (2005), o tipo climático da região, segundo a classificação de Koeppen, corresponde a "Cwa": as temperaturas médias mensais variam de $17,6^{\circ} \mathrm{C}$ (no mês mais frio, julho) a $23,5^{\circ} \mathrm{C}$ (no mês mais quente, fevereiro). A precipitação media anual é de $1.478 \mathrm{~mm}$, enquanto a evaporação potencial é de $992,67 \mathrm{~mm}$ e a evaporação real é de 919,1 mm. A área apresenta uma estrutura onde os elementos climáticos - temperatura, umidade do ar, precipitação, radiação solar e vento -são relativamente homogêneos em toda sua extensão. Na região, alguns tipos de precipitação são particularmente importantes: (1) chuvas convectivas, que ocorrem tipicamente de setembro a abril, e (2) chuvas frontais, que ocorrem em qualquer época do ano, com maior freqüência no outono e no inverno. Rocha et al (2005) caracterizam o clima local como chuvoso de temperaturas el evadas e inverno ameno.

No cerrado, o processo de evapotranspiração apresenta notável amplitude sazonal, variando de $6 \mathrm{~mm} \cdot \mathrm{dia}^{-1}$ (estação chuvosa, que compreende principalmente os meses de dezembro, janeiro e fevereiro) até $1 \mathrm{~mm} \cdot$ dia-1 $^{-1}$ (estação seca, que compreende principalmente os meses de junho, julho e agosto). Rocha et al (2005) comentam que a oscila- 
Tabela 1 -Descrição das variáveis coletadas nos sítios experimentais de cerrado s.s. e de cana-de-açúcar. Fonte: Juárez (2004).

\begin{tabular}{|c|c|c|c|c|c|}
\hline \multirow{2}{*}{ Variável } & \multirow{2}{*}{$\begin{array}{l}\text { Símbolo } \\
\text { (Unidade) }\end{array}$} & \multicolumn{2}{|c|}{ Sensor } & \multicolumn{2}{|c|}{ Posição (m) } \\
\hline & & Cerrado s.s. & Cana-de-Açúcar & Cerrado s.s. & Cana-de-Açúcar \\
\hline Temperatura do ar & $\mathrm{T}\left({ }^{\circ} \mathrm{C}\right)$ & $\begin{array}{c}\text { Psicrometro CSI } \\
\text { H M P45C }\end{array}$ & $\begin{array}{c}\text { Psicrometro CSI } \\
\text { H M P45C }\end{array}$ & 21 & 7 \\
\hline Umidade relativa & UR (\%) & $\begin{array}{c}\text { Psicrometro CSI } \\
\text { H M P45C }\end{array}$ & $\begin{array}{c}\text { Psicrometro CSI } \\
\text { H M P45C }\end{array}$ & 21 & 7 \\
\hline Pressão Atmosférica & Press ( $\mathrm{hPa}$ ) & Vaisalla & Vaisalla & 21 & 7 \\
\hline Velocidade do vento & $U\left(m s^{-1}\right)$ & RM Young & RM Young & 21 & 7 \\
\hline $\begin{array}{l}\text { Irradiância solar global } \\
\text { incidente }\end{array}$ & $\mathrm{Ki}\left(\mathrm{W} \cdot \mathrm{m}^{-2}\right)$ & LiCor 200X & LiCor 200X & 21 & 7 \\
\hline Saldo de radiação & $\operatorname{Rn}\left(\mathrm{W} \cdot \mathrm{m}^{-2}\right)$ & REBS & REBS & 21 & 7 \\
\hline Umidade do solo & $W_{1}\left(m^{3} m^{-3}\right)$ & CSI 615 & CSI 615 & $-0,1 \mathrm{~m}$ & $-0,1 \mathrm{~m}$ \\
\hline Temperatura do solo & $\mathrm{T}_{10}\left({ }^{\circ} \mathrm{C}\right)$ & CSI 108 & CSI 108 & $-0,1 \mathrm{~m}$ & $-0,1 \mathrm{~m}$ \\
\hline Fluxo de calor sensível & $\mathrm{H}\left(\mathrm{W} \cdot \mathrm{m}^{-2}\right)$ & Eddy covariance & Eddy covariance & 21 & 7 \\
\hline Fluxo de calor latente & $\operatorname{LE}\left(\mathrm{W} \cdot \mathrm{m}^{-2}\right)$ & Eddy covariance & Eddy covariance & 21 & 7 \\
\hline
\end{tabular}

ção anual é forcada basicamente por dois controles: (1) a variação da oferta de energia, que apresenta o mesmo padrão da evapotranspiração (máximos no verão e mínimos no inverno) e (2) as condições da vegetação e da umidade disponível no solo. A vegetação perde parte significativa das folhas verdes nos estratos arbóreo e herbáceo, reduzindo assim a capacidade de transpiração. A umidade do solo, que também passa por um mínimo no inverno, também contribui para que a componente de evaporação do solo se reduza e, principalmente, para que o acesso a umidade nos baixos níveis seja menor, o que limita a extração de água do estrato herbáceo. Porém, a existência de uma pequena taxa de evapotranspiração na estação seca é um importante indicador que parte da vegetação continua ativa, do ponto de vista biológico.

Em áreas de cana-de-açúcar, a evapotranspiração depende também do ciclo da cultura, uma vez que ocorrem variações significativas da estrutura da vegetação, passando de condições de solo descoberto ( período de plantação e/ ou colheita) a cultivos plenamente desenvolvidos. Para o ano de 1998, Cabral et al (2003) apresentam dados de evapotranspiração que variam de $0,5 \mathrm{~mm} \cdot \mathrm{dia}^{-1}$ ( na estação seca) a 6,7 mm.dia-1 (na estação chuvosa), correspondendo à transpiração das plantas e à evaporação da chuva interceptada pelo dossel.

\section{SÍTIOS DE MONITORAMENTO DOS FLUXOSTURBULENTOS}

O conjunto instrumental foi instalado em duas torres a uma altura de 7 metros em plantações de cana-de-açúcar, nas coordenadas $21^{\circ} 10^{\prime} \mathrm{S}$ e $48^{\circ} 06^{\prime}$, e 21 metros em áreas de cerrado sensu stricto, nas coordenadas $21^{\circ} 37^{\prime} \mathrm{S}$ e $47^{\circ} 37^{\prime} \mathrm{W}$. Foram coletadas medidas de variáveis do clima (ar e solo), de fluxos radiativos, atmosféricos e turbulentos, amostradas a cada 15 segundos e gravadas em médias de 30 minutos em um data logger Campbell CR10X. A tabela 1 apresenta as descrições das variáveis coletadas nos sítios experimentais do cerrado e cana. Juárez (2004) apresenta descrições detalhadas dos processos de medição dos fluxos turbulentos e de energia na área de estudo.

\section{ANÁLISE DAS COMPONENTES PRINCIPAISE CLUSTER}

Para identificar quais as variáveis do balanço de energia são mais importantes para a evapotranspiração, utilizou-se análise multivariada através da técnica de componentes principais (PCA) e agrupamento de variáveis (clusters).

O método de análise por componentes principais procura encontrar um novo conjunto de 
variáveis que retenham o máximo de variância, através de uma combinação linear dos dados originais (Wilks 1995). A estimava das componentes principais é desenvolvida através da informação contida na matriz de covariância dos dados. Para a aplicação da técnica é necessária à padronização dos dados para que toda a série fique com a mesma grandeza de valores, neste caso foram calculadas as anomalias de cada uma das variáveis. 0 passo posterior é obter os autovetores que são os valores que representam os pesos de cada uma das variáveis em cada componente (eixos) e funcionam como coeficientes de correlação variando entre -1 e 1 e os autovalores que representam a contribuição relativa de cada componente para explicar a variação total dos dados (Gomes et al, 2004). O número de eixos ou componentes podem se igualar ao número de variáveis, porém os eixos posteriores vão contribuir cada vez menos para explicar os dados (Kent e Coker 1992). Para facilitar a visualização dos resultados serão utilizados gráficos em duas dimensões.

Para verificar o agrupamento entre as variáveis foi utilizada a análise de clusters que apresentam a vantagem de reduzirem o espaço multidimensional a uma medida de distância entre os objetos, sendo esta representada em um espaço bidimensional, muito mais simplificado do que o espaço multidimensional (Mardia et al 1995). A análise de cluster busca agrupar elementos de dados baseando-se na similaridade entre eles. Os grupos são determinados de forma a obter-se homogeneidade dentro dos grupos e heterogeneidade entre eles. Como resultado da análise de agrupamento, tem-se o dendograma, que apresenta o arranjo entre os objetos em uma escala de distância. Este arranjo indica apenas afinidade entre os grupos, não definindo nenhuma ordenação entre estes. As distâncias são medidas e utilizadas para a representação dos pontos na estrutura de similaridade, representando o menor espaço entre dois pontos, sendo utilizada neste trabalho a medida da distância euclidiana entre dois vetores $i, j$, de acordo com a equação 1 .

$d_{i, j}=\left[\sum_{k=1}^{K} w_{k}\left(x_{k, i}-x_{k, j}\right)^{2}\right]^{1 / 2}$

Depois de calcular a distância é utilizado o método aglomerativo, onde cada elemento inicia-se representando um grupo, e a cada passo, um grupo ou elemento é ligado a outro de acordo com sua similaridade, até o último passo, onde é formado um grupo único com todos os elementos. Utilizou-se o método de ligação completa (complete linkage) que emprega a distância máxima tendo a tendência de formar grupos compactos, nos quais os ruídos demoram a ser incorporados nos grupos.

\section{RESULTADOSE DISCUSSÕES}

A figura 2 apresenta a variabilidade sazonal da temperatura média diária, umidade relativa do ar, velocidade do vento, pressão atmosférica, temperatura e umidade do solo a $10 \mathrm{~cm}$ de profundidade, além da irradiância solar incidente e do saldo de radiação e dosfluxos de calor sensível e latente, para os anos de 2001 e 2002. Essas variáveis apresentam um ciclo sazonal definido de acordo com as estações inverno e verão. No cerrado e em áreas de cana-deaçúcar, a variação da temperatura média diária oscilou entre $18^{\circ} \mathrm{C}$ na estação seca e $26^{\circ} \mathrm{C}$ na estação chuvosa, enquanto na área de cana a variação foi de temperaturas médias menores que $15^{\circ} \mathrm{C}$, principalmente entre maio e setembro, as quais estão associadas ao deslocamento de frentes frias polares. A variação sazonal da temperatura do ar repercute também na temperatura da superfície do solo.

Segundo Juárez (2004), essa variação é perceptível até $20 \mathrm{~cm}$ de profundidade. A temperatura do solo a $10 \mathrm{~cm}$ de profundidade apresenta mínimos de $14^{\circ} \mathrm{C}$ na estação seca e máximos de $27^{\circ} \mathrm{C}$ na estação chuvosa. A umidade relativa média do ar e do solo, bem como a pressão atmosférica, seguem a variabilidade sazonal da precipitação, diminuindo durante a estação seca e aumentando durante a estação chuvosa.

A umidade do solo, segundo Juárez (2004), está diretamente relacionada com a precipitação. Os períodos referentes à estação seca são facilmente reconhecidos pelo baixo teor de umidade do solo, enquanto que as precipitações produzem alterações significativas nesses índices de umidade. Para áreas de cerrado, a umidade varia entre 0,05 e $0,2 \mathrm{~m}^{3} \cdot \mathrm{m}^{-3}$, enquanto que em áreas de cultivo de cana-deaçúcar, essas variações ocorrem entre 0,3 e 0,5 $\mathrm{m}^{3} \cdot \mathrm{m}^{-3}$. Apesar de apresentar uma forte correlação sazonal, verificou-se que a umidade do solo em cultivos de cana é maior do que em áreas de cerrado. Isso pode ser explicado pelo processo de compactação das camadas superficiais, característica dos manejos 

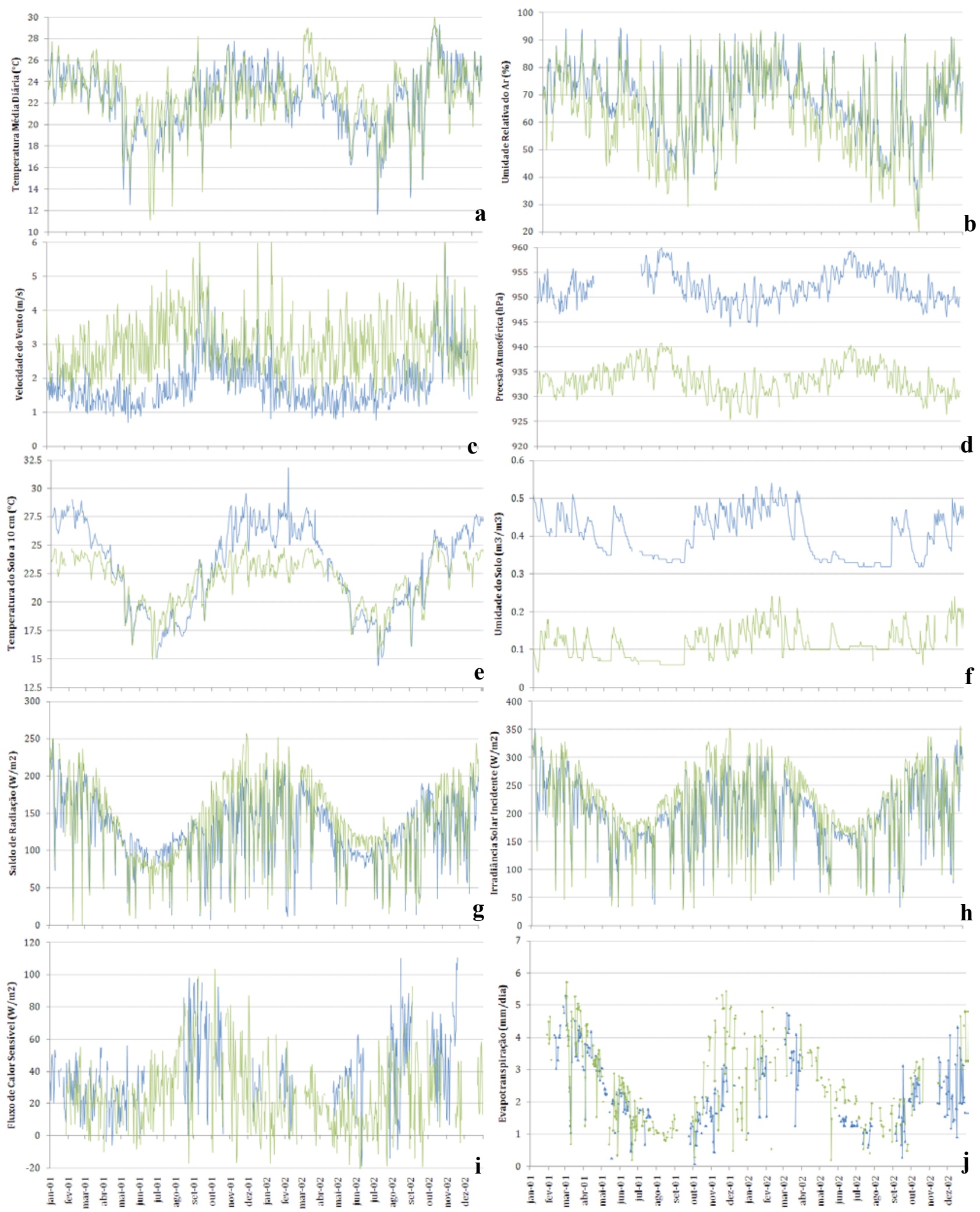

Figura 2 -Variação sazonal das médias diárias de temperatura (a), umidade relativa do ar (b), velocidade do vento (c), pressão atmosférica (d), temperatura do solo a $10 \mathrm{~cm}$ de prof undidade (e), umidade do solo a $10 \mathrm{~cm}$ de prof undidade (f), saldo de radiação (g), irradiância solar incidente (h), fluxo de calor sensível (i) e evapotranspiração (j) para áreas de canade-açúcar (azul) e áreas de cerrado sensu stricto (verde). 
de lavouras de cana, e pela redução da atividade radicular nessas camadas, o que as mantêm em princípio mais úmidas (Juárez 2004). As variações da pressão atmosférica em áreas de cerrado e cana-deaçúcar estão associadas à altitude da torre de medição em relação ao nível do mar.

A velocidade média diária do vento está associada principalmente à sazonalidade da energia cinética da atmosfera no hemisfério sul, em grande parte devido à Zona de Convergência do Atlântico Sul (ZCAS), e secundariamente ao efeito combinado da queda das folhas, o que reduz a desaceleração do vento pelo dossel (Juárez 2004). No cerrado, as variações médias diárias do vento foram de 2 a 6 m.s 1 , enquanto que em áreas de cana-de-açúcar essas variações ocorreram entre 1,5 e $5 \mathrm{~m} \cdot \mathrm{s}^{-1}$.

Os ciclos sazonais de irradiância solar incidente e do saldo de radiação para áreas de cerrado e cana-de-açúcar têm mínimos na estação seca e máximos na estação chuvosa, coerentes com o processo de incidência de radiação solar sobre a superfície terrestre (Juárez 2004).

Da mesma forma, as variações sazonais de $\mathrm{Ki}$ e Rn se assemelham aos da temperatura do ar e do solo, ou seja, o aumento da temperatura está diretamente associada ao aumento de Rn e Ki. Em áreas de cerrado, as médias máximas de Ki variam entre $200 \mathrm{~W} \cdot \mathrm{m}^{-2}$ na estação seca e $350 \mathrm{~W} \cdot \mathrm{m}^{-2}$ na estação chuvosa, enquanto que a variação de $\mathrm{Rn}$ foi de 125 a $250 \mathrm{~W} \mathrm{~m}^{-2}$, para as mesmas estações. Para áreas de cana-de-açúcar, Ki apresenta uma amplitude ligeiramente superior ao do cerrado, variando entre 100 W. $\mathrm{m}^{-2}$ na estação seca e $350 \mathrm{~W} \cdot \mathrm{m}^{-2}$ na estação chuvosa, enquanto $\mathrm{Rn}$ apresenta médias entre 100 e 200 W. $\mathrm{m}^{-2}$, para as mesmas estações.

O fluxo de calor latente apresenta oscilação a qual é forçada, basicamente, pela variação da oferta de energia e pelas condições da vegetação e da umidade disponível no solo (Rocha 2002). O fluxo de calor sensível, por sua vez, não é controlado apenas pelo saldo de radiação, mas também pela cobertura do dossel (Juárez 2004). A senescência, causada principalmente pela baixa oferta de umidade na estação seca, reduz a atividade fotossisntética da cultura. Diminuindo-se o índice de vegetação, uma maior quantidade de energia fica disponível para ser convertida em fluxo de calor sensível. Dessa forma, as médias diárias máximas de calor sensível aparecem em agosto e setembro, com variações observadas entre 40 e 80 W.m-2, enquanto as médias diárias mínimas aparecem em março e abril, com variações observadas entre 10 e $40 \mathrm{~W} \cdot \mathrm{m}^{-2}$. A influência da alternância de estações produz efeitos consideráveis sobre a vegetação, alterando a rugosidade e a resis- tência da superfície, modificando o particionamento da energia disponível em calor e vapor d'água.

A relação entre as variáveis climáticas pode ser entendida a partir da análise multivariada das componentes principais. $\mathrm{N}$ as tabelas 2 e 3 são apresentados os autovetores de cada um dos modos das componentes principais, para as torres de medições instaladas em áreas de cerrado e cultivos de cana-deaçúcar, respectivamente.

Nas tabelas 2 e 3 observa-se que são necessárias três componentes principais para explicar $75 \%$ ou mais da variância das variáveis observadas, sendo concentradas assim em três dimensões as informações anteriormente diluídas em seis variáveis. A primeira componente explica $40,71 \%$ da variância total das variáveis em áreas de cerrado e 45,51\% em áreas de cultivos de cana-de-açúcar. A segunda componente explica $23,84 \%$ e $22,75 \%$ da variância total das variáveis em áreas de cerrado e cana-de-açúcar, respectivamente, enquanto a terceira componente explica $10,40 \%$ e $14,73 \%$ da variância total das variáveis em áreas de cerrado e cana-de-açúcar, respectivamente.

A primeira componente representa as principais características do processo de aporte de energia e aquecimento da superfície terrestre, destacando-se as variáveis $K i, R n, T, T_{10}$ e LE, que apresentam variações no mesmo sentido, ou seja, com o aumento de disponibilidade de energia, representado por $\mathrm{Ki}$ e Rn, ocorrem aumentos da temperatura do solo e do ar e no fluxo de calor latente (evapotranspiração). Esse mesmo comportamento é verificado sobre áreas de cerrado e de cultivos de cana-de-açúcar.

A segunda componente é representada por variáveis que apresentam comportamento inverso e que estão relacionadas com o processo de circulação atmosférica local e com a precipitação. Com a diminuição da pressão atmosférica e da velocidade dos ventos, ocorre um aumento significativo da umidade do ar e do solo, ou seja, a redução da pressão atmosférica está inversamente relacionada com a precipitação, que condiciona esses índices de umidade. Esse padrão é verificado principalmente em áreas de cerrado. Em áreas de cultivos de cana-de-açúcar, a velocidade do vento perde sua importância significativa na segunda componente, uma vez que nessas áreas há um aumento da rugosidade da superfície, dificultando o processo de circulação dos ventos em superfície. As figuras 3 e 4 apresentam a interrelação das variáveis climáticas nas duas componentes principais, em áreas de cerrado e cana-de-açúcar, respectivamente. 
T abela 2 - Resultados dos autovetores e das variâncias para áreas de cerrado.

\begin{tabular}{llll}
\hline Variáveis Climáticas & Modo 1 & Modo 2 & Modo 3 \\
\hline T & -0.379 & 0.058 & 0.524 \\
UR & 0.094 & -0.532 & -0.240 \\
U & 0.073 & 0.332 & -0.071 \\
Press & 0.267 & 0.404 & -0.037 \\
Kn & -0.426 & 0.240 & -0.239 \\
$\mathrm{Rn}$ & -0.452 & 0.057 & -0.264 \\
$\mathrm{H}$ & -0.149 & 0.401 & -0.506 \\
$\mathrm{LE}$ & -0.427 & -0.042 & -0.015 \\
$\mathrm{~T}_{10}$ & -0.415 & -0.173 & 0.260 \\
$\mathrm{~W}_{1}$ & -0.089 & -0.433 & -0.460 \\
\hline Variância (\%) & 40.71 & 23.84 & 10.40 \\
Variância acumulada (\%) & 40.71 & 64.55 & 74.95 \\
\hline
\end{tabular}

Tabela 3 - Resultados dos autovetores e das variâncias para áreas de cultivos de cana-de-açúcar.

\begin{tabular}{llll}
\hline Variáveis Climáticas & Modo 1 & Modo 2 & Modo 3 \\
\hline T & -0.414 & 0.062 & -0.152 \\
UR & 0.073 & -0.609 & 0.067 \\
U & -0.088 & 0.076 & -0.685 \\
Press & 0.269 & 0.276 & 0.371 \\
Kn & -0.415 & 0.209 & 0.200 \\
Rn & -0.421 & 0.150 & 0.250 \\
$H$ & -0.261 & 0.447 & -0.184 \\
LE & -0.336 & -0.145 & 0.457 \\
$\mathrm{~T}_{10}$ & -0.384 & -0.301 & -0.122 \\
W & -0.256 & -0.408 & -0.078 \\
\hline Variância (\%) & 45.51 & 22.75 & 14.73 \\
Variância acumulada (\%) & 45.51 & 68.26 & 82.99 \\
\hline
\end{tabular}

A terceira componente está relacionada às condições da vegetação. Em áreas de cerrado, as variáveis climáticas que mais se destacam são temperatura do ar e fluxo de calor sensível, que variam inversamente. A alternância de estações secas e chuvosas produz modificações significativas na estrutura da vegetação. Com a redução da atividade fotossintética da vegetação, que ocorre durante a estação seca e amena, uma maior quantidade de energia fica disponível para ser convertida em fluxo de calor sensível. Para áreas de cana-de-açúcar, o comportamento das variáveis climáticas é alterado em função do estágio de desenvolvimento da cultura, principalmente durante o período de plantio e de corte. Assim, a velocidade do vento e o fluxo de calor latente/ evapotranspiração destacam-se nessa terceira componente, uma vez que durante o processo de plantio e/ ou corte, há um aumento na velocidade do vento e conseqüentemente uma redução no processo de evapotranspiração.

0 agrupamento das variáveis através de similaridade em áreas de cerrado apresentou a formação de três grupos principais: (1) Ki, Rn, LE, T, $T_{10}$ e H ; (2) UR e $W_{1}$; e, (3) U e pressão atmosférica. Em áreas de cana-de-açúcar, também se formaram três grupos principais: (1) Ki, Rn e LE; (2) T, T 10 , UR e $\mathrm{W}_{1}$; e, (3) U, H e pressão atmosférica. O agrupamento das variáveis climáticas confirma os resultados das análises das componentes principais. A figura 5 apresenta o agrupamento das variáveis climáticas coletadas em áreas de cerrado (a) e cana-deaçúcar (b). 


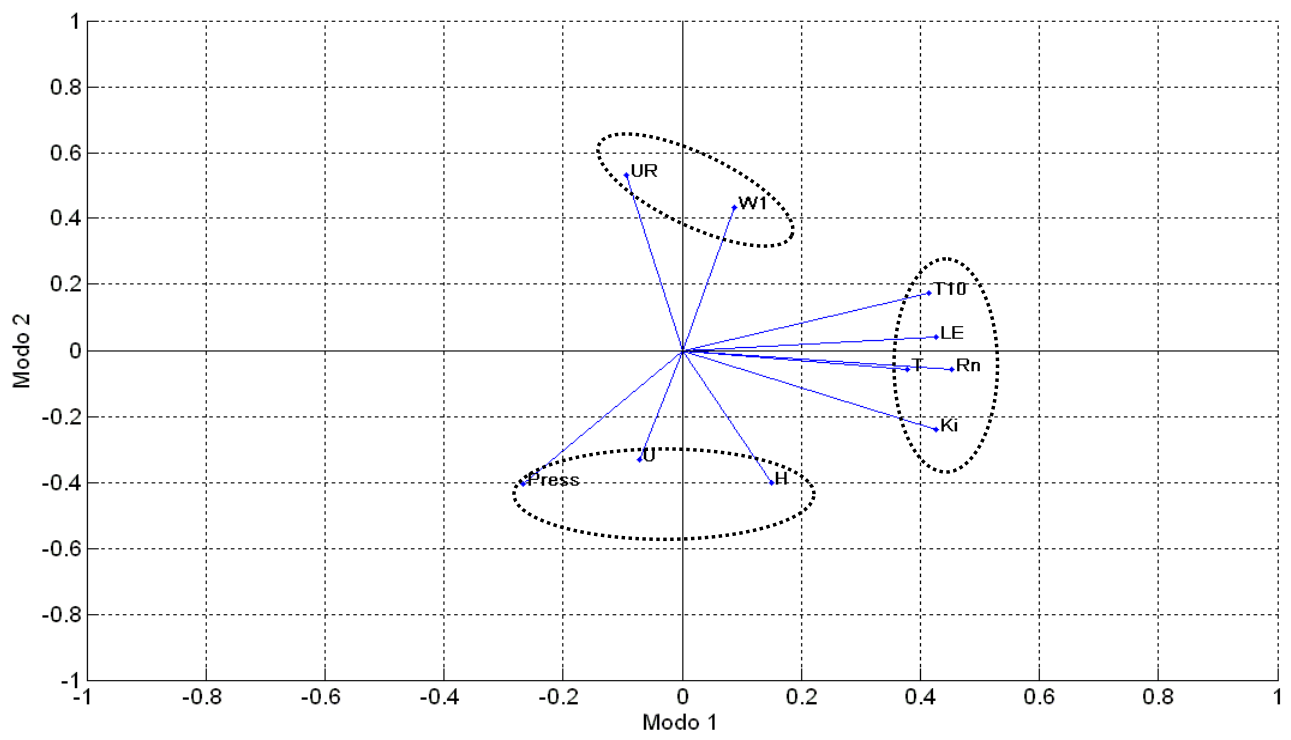

Figura 3 -Primeiro e segundo modos das componentes principais das variáveis climáticas em áreas de cerrado.

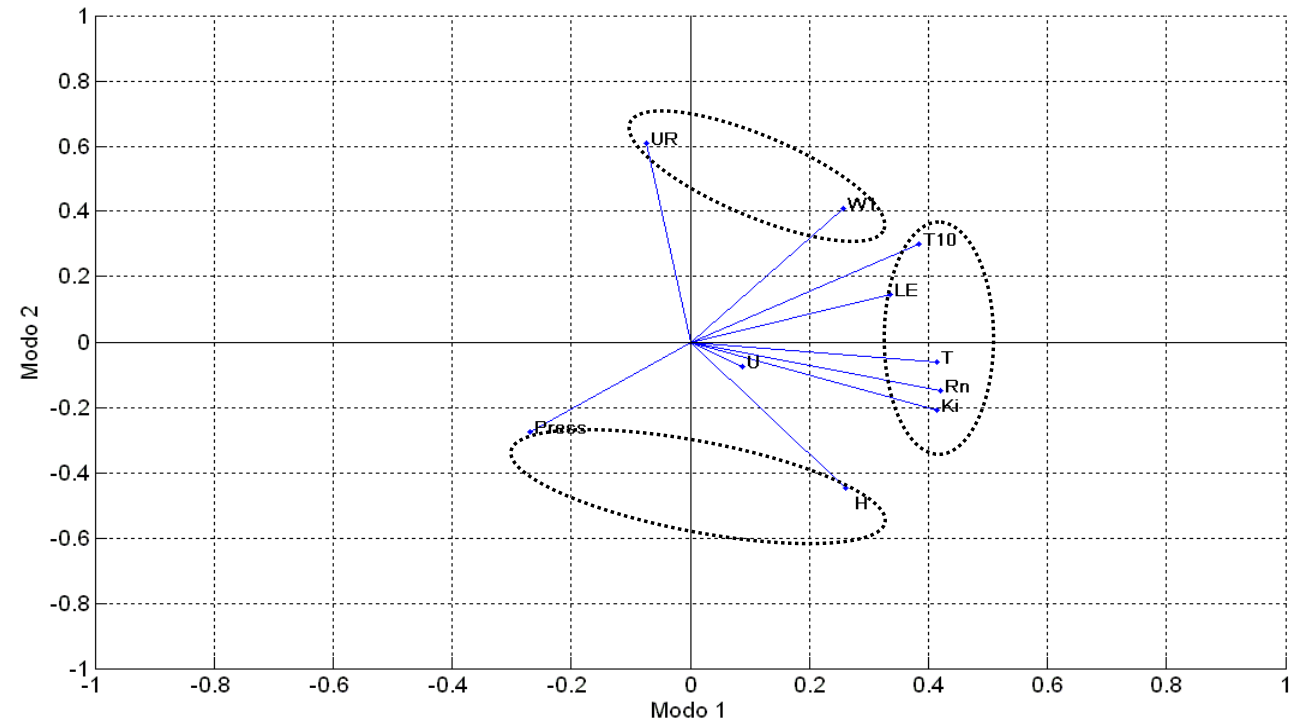

Figura 4 -Primeiro e segundo modos das componentes principais das variáveis climáticas em áreas de cana-de-açúcar.
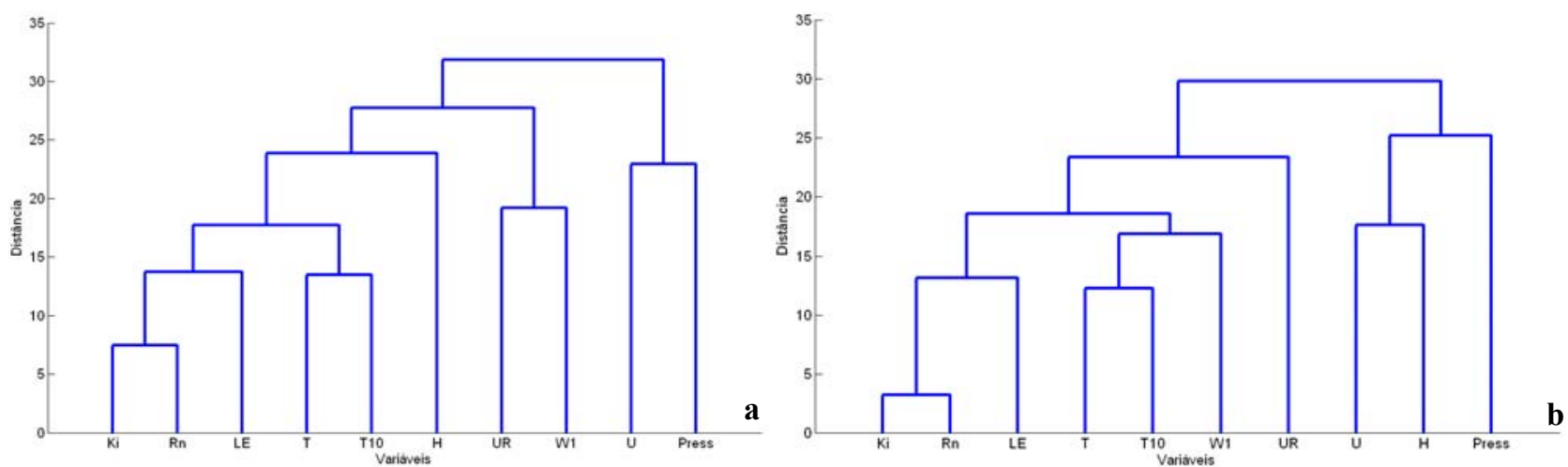

Figura 5 -Agrupamento das variáveis climáticas coletadas em áreas de cerrado (a) e cana-de-açúcar (b). 
CONSIDERAÇÕES FINAIS

A importância das variáveis climática envolvidas no processo de evapotranspiração foi avaliada a partir das técnicas de estatística multivariada Análise de Componentes Principais e Análise de Agrupamentos. As técnicas foram aplicadas a dados coletados durante os anos de 2001 e 2002 em dois sítios experimentais localizados em remanescentes de cerrado sensu stricto e cultivos de cana-de-açúcar. Assim, concluiu-se que o processo de evapotranspiração, para a área estudada, é comandada por três componentes, que explicam 75 e $82 \%$ da variância dos dados em áreas de cerrado e cultivos de cana-deaçúcar, respectivamente: (1) variação da oferta de energia, representada principalmente pelas variáveis Ki e Rn, que explicam $40,71 \%$ da variância do processo em áreas de cerrado e 45,51\% em áreas de cana-de-açúcar; (2) processo de circulação atmosférica local, representada pelas variáveis de velocidade do vento e pressão atmosférica, que regulam o sistema de precipitação e as condições de umidade do ar e do solo, que explicam $23,84 \%$ da variância do processo em áreas de cerrado e $22,75 \%$ em áreas de cana-de-açúcar; e, (3) condições da vegetação, que apresentam modificações significativas com a alternância de estações secas e úmidas, explicando $10,40 \%$ da variância do processo em áreas de cerrado e $14,73 \%$ em áreas de cana-de-açúcar.

Algumas alterações significativas no processo de evapotranspiração podem ser observadas em áreas de cultivo de cana-de-açúcar, principalmente relacionadas com o estágio de desenvolvimento da cultura (plantio, crescimento e colheita). Com a alteração na estrutura do dossel, a rugosidade dessa superfície é alterada, acarretando em mudanças significativas na velocidade do vento. Além disso, a compactação das camadas superficiais do solo também altera a disponibilidade de umidade no perfil do solo. Essas modificações, que apresentam significância na segunda componente, respondendo por aproximadamente $23 \%$ da variância dos dados, repercutem consideravelmente no processo de evapotranspiração, alterando o particionamento dos fluxos de calor sensível e latente.

\section{AGRADECIMENTOS}

Os autores agradecem ao CNPq pela concessão de bolsa de doutorado ao primeiro e segundo autores.

\section{REFERÊNCIAS}

ALLEN, RG; PEREIRA, LS; RAES, D; SMITH, M. 1998. Crop evapotranspiration-Guidelines for computing crop water requirements. Irrigation and drainage paper FAO-56. Water Resources, Development and Management Service, Roma.

BATALHA, MA 1997. Análise da vegetação da ARIE Cerrado Pé de Gigante (Santa Rita do Passa Quatro, SP). Dissertação de Mestrado em Ecologia. Universidade de São Paulo.

CABRAL, OMR.; da ROCHA, HR; LIGO, MA; BRUNINI, O; DIAS, MAFS. 2003. Fluxos turbulentos de calor sensível, vapor d'água e CO2 sobre plantação de canade-açúcar (Saccharum sp) em Sertãozinho, SP. Revista Brasileira de Meteorologia, v. 18, n. 1, p. 61-70.

COLLISCHONN, W. Simulação hidrológica de grandes bacias. 2001. Tese de Doutorado em Recursos Hídricos e Saneamento Ambiental. Universidade Federal do Rio Grande do Sul.

COLLISCHONN, W; TUCCI, CEM. 2001. Simulação hidrológica de grandes bacias. Revista Brasileira de Recursos Hídricos, v. 6, n. 1.

FURLEY, PA. 1999. The nature and diversity of neotropical savanna vegetation with particular reference to the Brazilian cerrados. Global Ecology and Biogeography, v. 8, p. 223-241.

GOMES, JBV; CURI, N; MOTTA, PEF; KER, JC; MARQUES, JJGSM; SCHULZE, DG. 2004. Análise de componentes principais de atributos físicos, químicos e mineralógicos de solos do bioma cerrado. Revista Brasileira de Ciência do Solo, v. 28, n. 1, p. 137-153.

JUÁREZ, RIN. 2004. Variabilidade climática regional e controle da vegetação no sudeste: Um estudo de observações sobre cerrado e cana de açúcar e modelagem numérica da atmosfera. Tese de Doutorado em Ciências Atmosféricas. Universidade de São Paulo.

KENT, M; COKER, P. 1992. Vegetation description and analysis. Baffins Lane, John Wiley \& Sons, 363p.

KUMAR, M; RAGHUWANSHI, NS; SINGH, R; WALLENDER, WW; PRUITT, WO. 2002. Estimating evapotranspiration using artificial neural network. Journal of Irrigation and Drainage Engineering, v.128, n.4, p.224233.

MARDIA, KV; KENT, JT; BIBBY, JM. 1995. Multivariate analysis. London: Academic Press, 518p.

ROCHA, HR; JUÁREZ, RIN; LIGO, M; CABRAL, OMR; MESQUITA, H; MITTENCOURT, M. 2004. Ciclo hidrológico e microclima. In PIVELLO, VR; VARANDA, EM. 2005. O cerrado pé de gigante: ecologia e conservação - Parque Estadual da Vassununga. São Paulo: Secretaria do Meio Ambiente. 
ROCHA, HR; ROSOLEM, R; JUAREZ; RIN, TANNUS, RN; LIGO, MA; CABRAL, OMR; DIAS, MAFS. 2002. Measurements of $\mathrm{CO} 2$ exchange over a woodland savanna Cerrado Sensu stricto) in southeast Brasil. Biota Neotropica, v.2, n. 1, 11p.

RUHOFF, AL; JUÁREZ, RIN; FREITAS, RM; COLLISCHONN, W; ROCHA, HR. 2009. Variabilidade dos fluxos de calor sensivel e latente em áreas de cerrado sensu stricto. Simpósio Brasileiro de Sensoriamento Remoto. São José dos Campos, SP: INPE, p. 4829-4836.

2008. Estimativa da sazonalidade da evapotranspiração em plantação de cana-de-açúcar (saccharum sp.) a partir de dados do sensor Terra/MODIS. II Simpósio de Recursos Hídricos do SulSudeste, Rio de Janeiro, RJ: ABRH. 20 p.

WILKS, DS. 1995: Statistical Methods in the Atmospheric Sciences: an introduction. International Geophysics Series, Academic Press, v. 59, 464 p.

\section{Multivariate Analysis of the Evapotranspiration Process in Cerrado and SugarcaneAreas}

\section{ABSTRACT}

One of the most important variables in the hydrological cycle is evapotranspiration, which contributes to the connection between energy, climate and water availability. It is considered a very complex phenomenon, for it depends on the interaction between several climate variables to identify the behavior of each climate variable and to assess factors which highly influence the evapotranspiration process. A set of samples of climate variables was analyzed. They were collected at two turbulent vortex monitoring sites installed in sugar cane fields and remaining "cerrado" sensu stricto in the Grande River basin, São Paulo, Brazil. Therefore, multivariate statistical techniques for analyzing main components and for grouping variables were applied. It was concluded that the evapotranspiration process consists of three components which explain 75 and $82 \%$ of variance data in "cerrado" and sugar cane fields, respectively: (1) variation of energy supply, represented mainly by $\mathrm{Ki}$ and $\mathrm{Rn}$ variables; (2) local atmospheric circulation process, represented by wind speed and atmospheric pressu re variables, ruling the system of precipitation and air humidity and soil moisture, and; (3) conditions of the vegetation presenting significant changes in dry and humid seasons. Keywords: Multivariate statistics, Evapotranspiration, "Cerrado", Sugar Cane 